\title{
Fixed Point Theorems for Two Pairs of Nonself Mappings in Metrically Convex Spaces by Altering Distances
}

\author{
MOHD IMdAD AND LADLAY KHAN
}

\begin{abstract}
Some common fixed point theorems for two pairs of nonself mappings in complete metrically convex metric spaces are proved by altering distances between the points which generalize earlier results due to Khan et al. [15], Khan and Bharadwaj [14], Bianchini [5], Chatterjea [6] and others. Some related results are also discussed besides furnishing an illustrative example.
\end{abstract}

\section{INTRODUCTION}

The existing literature of fixed point theory contains a variety of results. Among them one variety is of those results which are proved by altering distances between the points. Delbosco [7] and Skof [21] obtained fixed point theorems for self maps on complete metric spaces by altering distances between the points with the aid of increasing continuous function. Khan et al. [16] improved some fixed point theorems of Rakotch [18], Reich [19] and Fisher [8] in a complete metric space which has been generalized and improved in several ways in recent years. Assad $[2,3]$ generalized a result due to Khan et al. [16] for nonself mappings in complete metrically convex metric spaces. Recently, Abdalla and Zaheer [4] partially extended the result due to Khan et al. [16] to a pair of nonself mappings employing the notion of weak commutativity due to Hadžić [9] which generalize earlier results due to Khan et al. [16], Assad [2,3] and some others.

The aim of this paper is to extend a result of Khan et al. [15] for two pairs of nonself mappings by altering distances which generalizes earlier results due to Khan et al. [15], Khan and Bhardwaj [14], Bianchini [5], Chatterjea [6] and others. For the sake of completeness, we state the following theorem due to Khan et al. $[15]$.

Theorem 1.1. Let $(X, d)$ be a complete metrically convex metric space and $K$ a nonempty closed subset of $X$. Let $T: K \rightarrow X$ be a mapping satisfying the

2000 Mathematics Subject Classification. Primary: 54H25; Secondary: $47 \mathrm{H} 10$.

Key words and phrases. Metrically convex metric space, weak commutativity, compatible mappings; coincidentally commuting mappings, complete metric spaces. 
inequality:

$$
d(T x, T y) \leq a \max \{d(x, T x), d(y, T y)\}+b\{d(x, T y)+d(y, T x)\}
$$

for every $x, y \in K$, where $a$ and $b$ are non-negative reals such that

$$
\begin{gathered}
\max \left\{\frac{a+b}{1-b}, \frac{b}{1-a-b}\right\}=h>0, \\
\max \left\{\frac{1+a+b}{1-b} h, \frac{1+b}{1-a-b} h\right\}=h^{\prime}, \\
\max \left\{h, h^{\prime}\right\}=h^{\prime \prime}<1 .
\end{gathered}
$$

Further, if for every $x \in \delta K, T x \in K$. Then $T$ has a unique fixed point in $K$.

\section{Preliminaries}

We need the following definitions and a lemma for subsequent discussion.

Definition 2.1 ([10]). Let $K$ be a nonempty subset of a metric space $(X, d)$ and $F, T: K \rightarrow X$. The pair $(F, T)$ is said to be weakly commuting if for every $x, y \in K$ with $x=F y$ and $T y \in K$ such that

$$
d(T x, F T y) \leq d(T y, F y) .
$$

Notice that for $K=X$, this definition reduces to that of Sessa [20].

Definition $2.2([9])$. Let $K$ be a nonempty subset of a metric space $(X, d)$ and $F, T: K \rightarrow X$. The pair $(F, T)$ is said to be compatible if for every sequence $\left\{x_{n}\right\} \subset K$ and from the relation

$$
\lim _{n \rightarrow \infty} d\left(F x_{n}, T x_{n}\right)=0
$$

and $T x_{n} \in K$ (for every $n \in N$ ), it follows that

$$
\lim _{n \rightarrow \infty} d\left(T y_{n}, F T x_{n}\right)=0
$$

for every sequence $y_{n} \in K$ such that $y_{n}=F x_{n}, n \in N$.

Notice that for $K=X$, this definition reduces to that of Jungck [12].

Definition 2.3 (11). Let $K$ be a nonempty subset of a metric space $(X, d)$ and $F, T: K \rightarrow X$. The pair $(F, T)$ is said to be pointwise $R$-weakly commuting on $K$ if for given $y \in K$, there exists a real number $R>0$ such that

$$
d(T F y, F T y) \leq R(y) d(T y, F y)
$$

provided that $T y, F y \in K$.

The pair $(F, T)$ will be called $R$-weakly commuting on $K$ if $(2.3 .1)$ holds for all $y \in K$ with some $R>0$. 
Notice that for $R=1$ in Definition 2.3, we get the definition of weak commutativity on $K$ due to Hadžić and Gajić [10] whereas for $R=1$ and $K=X$ the weak commutativity due to Sessa [20]. Also by setting $K=X$, we get the definitions of pointwise $R$-weak commutativity and $R$-weak commutativity due to Pant [17]. Here, it is worth noting that compatible maps are necessarily pointwise $R$-weakly commuting because compatible maps commute at coincidence points. However, pointwise $R$-weakly commuting pairs need not be compatible.

Definition 2.4 ([11]). A pair of nonself mappings $(F, T)$ defined on a nonempty subset $K$ of a metric space $(X, d)$ is said to be coincidentally commuting if $T x, F x \in K$ with $T x=F x$ implies that $F T x=T F x$.

Notice that for $K=X$, this definition reduces to the corresponding definition due to Jungck and Rhoades [13] for self mappings.

Definition 2.5. Let $(X, d)$ be a metric space and $K$ a nonempty subset of $X$. Let $F, G, S, T: K \rightarrow X$ be the mappings which satisfy the condition:

$$
\begin{aligned}
\phi(d(F x, G y)) \leq a \max \left\{\frac{1}{2} \phi\right. & (d(T x, S y)), \phi(d(T x, F x)), \phi(d(S y, G y))\}+ \\
& +b\{\phi(d(T x, G y))+\phi(d(S y, F x))\}
\end{aligned}
$$

for all $x, y \in K$ with $x \neq y, a, b \geq 0$ such that $a+2 b<1$ and $\phi: R^{+} \rightarrow R^{+}$be an increasing continuous function for which following properties hold:

$$
\phi(t)=0 \Leftrightarrow t=0 \quad \text { and } \quad \phi(2 t) \leq 2 \phi(t) .
$$

Then $(F, G)$ is called generalized $(T, S)$ contraction mapping of $K$ into $X$.

Definition $2.6([1])$. A metric space $(X, d)$ is said to be metrically convex if for any $x, y \in X$ with $x \neq y$ there exists a point $z \in X, x \neq z \neq y$ such that

$$
d(x, z)+d(z, y)=d(x, y) .
$$

Lemma 2.1 ([1]). Let $K$ be a nonempty closed subset of a metrically convex metric space $X$. If $x \in K$ and $y \notin K$, then there exists a point $z \in \delta K$ (the boundary of $K$ ) such that

$$
d(x, z)+d(z, y)=d(x, y)
$$

\section{Main Result}

Our main result runs as follows:

Theorem 3.1. Let $(X, d)$ be a complete metrically convex metric space and $K a$ nonempty closed subset of $X$. If $(F, G)$ is generalized $(T, S)$ contraction mapping of $K$ into $X$ satisfying:

(i) $\delta K \subseteq S K \cap T K, F K \cap K \subseteq S K, G K \cap K \subseteq T K$;

(ii) $T x \in \delta K \Rightarrow F x \in K, S x \in \delta K \Rightarrow G x \in K$ with $T K$ and $S K$ (or $F K$ and $G K)$ are closed subspaces of $X$. Then 
(a) $(F, T)$ has a point of coincidence;

(b) $(G, S)$ has a point of coincidence.

Moreover, if the pairs $(F, T)$ and $(G, S)$ are coincidentally commuting, then $F, G, S$ and $T$ have a unique common fixed point.

Proof. Firstly, we proceed to construct two sequences $\left\{x_{n}\right\}$ and $\left\{y_{n}\right\}$ in the following way.

Let $x \in \delta K$. Then (due to $\delta K \subseteq T K$ ) there exists a point $x_{0} \in K$ such that $x=T x_{0}$. Since $T x \in \delta K \Rightarrow F x \in K$, one concludes that $F x_{0} \in F K \cap K \subseteq S K$. Let $x_{1} \in K$ be such that $y_{1}=S x_{1}=F x_{0} \in K$. Since $y_{1}=F x_{0}$, there exists a point $y_{2}=G x_{1}$ such that

$$
d\left(y_{1}, y_{2}\right)=d\left(F x_{0}, G x_{1}\right)
$$

Suppose $y_{2} \in K$. Then $y_{2} \in G K \cap K \subseteq T K$ which implies that there exists a point $x_{2} \in K$ such that $y_{2}=T x_{2}$. Otherwise, if $y_{2} \notin K$, then there exists a point $p \in \delta K$ such that

$$
d\left(S x_{1}, p\right)+d\left(p, y_{2}\right)=d\left(S x_{1}, y_{2}\right) .
$$

Since $p \in \delta K \subseteq T K$ there exists a point $x_{2} \in K$ with $p=T x_{2}$ so that

$$
d\left(S x_{1}, T x_{2}\right)+d\left(T x_{2}, y_{2}\right)=d\left(S x_{1}, y_{2}\right) .
$$

Let $y_{3}=F x_{2}$ be such that $d\left(y_{2}, y_{3}\right)=d\left(G x_{1}, F x_{2}\right)$.

Thus, repeating the foregoing arguments, one obtains two sequences $\left\{x_{n}\right\}$ and $\left\{y_{n}\right\}$ such that

(iii) $y_{2 n}=G x_{2 n-1}, y_{2 n+1}=F x_{2 n}$;

(iv) $y_{2 n} \in K \Rightarrow y_{2 n}=T x_{2 n}$ or $y_{2 n} \notin K \Rightarrow T x_{2 n} \in \delta K$ and

$$
d\left(S x_{2 n-1}, T x_{2 n}\right)+d\left(T x_{2 n}, y_{2 n}\right)=d\left(S x_{2 n-1}, y_{2 n}\right)
$$

(v) $y_{2 n+1} \in K \Rightarrow y_{2 n+1}=S x_{2 n+1}$ or $y_{2 n+1} \notin K \Rightarrow S x_{2 n+1} \in \delta K$ and

$$
d\left(T x_{2 n}, S x_{2 n+1}\right)+d\left(S x_{2 n+1}, y_{2 n+1}\right)=d\left(T x_{2 n}, y_{2 n+1}\right) .
$$

We denote

$$
\begin{aligned}
P_{\circ} & =\left\{T x_{2 i} \in\left\{T x_{2 n}\right\}: T x_{2 i}=y_{2 i}\right\}, \\
P_{1} & =\left\{T x_{2 i} \in\left\{T x_{2 n}\right\}: T x_{2 i} \neq y_{2 i}\right\}, \\
Q_{\circ} & =\left\{S x_{2 i+1} \in\left\{S x_{2 n+1}\right\}: S x_{2 i+1}=y_{2 i+1}\right\}, \\
Q_{1} & =\left\{S x_{2 i+1} \in\left\{S x_{2 n+1}\right\}: S x_{2 i+1} \neq y_{2 i+1}\right\} .
\end{aligned}
$$

Note that $\left(T x_{2 n}, S x_{2 n+1}\right) \notin P_{1} \times Q_{1}$ because if $T x_{2 n} \in P_{1}$, then $y_{2 n} \neq T x_{2 n}$ and one infers that $T x_{2 n} \in \delta K$ which implies that $y_{2 n+1}=F x_{2 n} \in K$. Hence $y_{2 n+1}=S x_{2 n+1} \in Q_{\circ}$. Similarly, one can argue that $\left(S x_{2 n-1}, T x_{2 n}\right) \notin Q_{1} \times P_{1}$.

Now, we distinguish the following three cases. 
Case 1. If $\left(T x_{2 n}, S x_{2 n+1}\right) \in P_{\circ} \times Q_{\circ}$, then

$$
\begin{aligned}
\phi\left(d\left(T x_{2 n}, S x_{2 n+1}\right)\right)= & \phi\left(d\left(F x_{2 n}, G x_{2 n-1}\right)\right) \leq \\
\leq & a \max \left\{\frac{1}{2} \phi\left(d\left(T x_{2 n}, S x_{2 n-1}\right)\right), \phi\left(d\left(T x_{2 n}, F x_{2 n}\right)\right),\right. \\
& \left.\phi\left(d\left(S x_{2 n-1}, G x_{2 n-1}\right)\right)\right\}+b\left\{\phi\left(d\left(T x_{2 n}, G x_{2 n-1}\right)\right)+\right. \\
& \left.\quad+\phi\left(d\left(F x_{2 n}, S x_{2 n-1}\right)\right)\right\}= \\
= & a \max \left\{\phi\left(d\left(y_{2 n}, y_{2 n-1}\right)\right), \phi\left(d\left(y_{2 n}, y_{2 n+1}\right)\right)\right\}+ \\
& \quad+b \phi\left(d\left(y_{2 n-1}, y_{2 n+1}\right)\right)= \\
= & a \max \left\{\phi\left(d\left(y_{2 n-1}, y_{2 n}\right)\right), \phi\left(d\left(y_{2 n}, y_{2 n+1}\right)\right)\right\}+ \\
& \quad+b \phi\left\{2 \max \left[d\left(y_{2 n-1}, y_{2 n}\right), d\left(y_{2 n}, y_{2 n+1}\right)\right]\right\} .
\end{aligned}
$$

If $d\left(y_{2 n-1}, y_{2 n}\right) \geq d\left(y_{2 n+1}, y_{2 n}\right)$, then

$$
\phi\left(d\left(T x_{2 n}, S x_{2 n+1}\right)\right) \leq(a+2 b) \phi\left(d\left(T x_{2 n}, S x_{2 n-1}\right)\right) .
$$

Otherwise, if $d\left(y_{2 n-1}, y_{2 n}\right) \leq d\left(y_{2 n+1}, y_{2 n}\right)$, then we have

$$
\begin{aligned}
\phi\left(d\left(T x_{2 n}, S x_{2 n+1}\right)\right) & \leq a \phi\left(d\left(y_{2 n}, y_{2 n+1}\right)\right)+2 b \phi\left(d\left(y_{2 n}, y_{2 n+1}\right)\right) \leq \\
& \leq(a+2 b) \phi\left(d\left(y_{2 n}, y_{2 n+1}\right)\right),
\end{aligned}
$$

which is a contradiction. Hence

$$
\phi\left(d\left(T x_{2 n}, S x_{2 n+1}\right)\right) \leq(a+2 b) \phi\left(d\left(T x_{2 n}, S x_{2 n-1}\right)\right) .
$$

Similarly, if $\left(S x_{2 n-1}, T x_{2 n}\right) \in Q_{\circ} \times P_{\circ}$, then one obtains

$$
\phi\left(d\left(S x_{2 n-1}, T x_{2 n}\right)\right) \leq(a+2 b) \phi\left(d\left(S x_{2 n-1}, T x_{2 n-2}\right)\right) .
$$

Case 2. If $\left(T x_{2 n}, S x_{2 n+1}\right) \in P_{\circ} \times Q_{1}$, then we have

$$
d\left(T x_{2 n}, S x_{2 n+1}\right)+d\left(S x_{2 n+1}, y_{2 n+1}\right)=d\left(T x_{2 n}, y_{2 n+1}\right),
$$

which in turn yields

$$
d\left(T x_{2 n}, S x_{2 n+1}\right) \leq d\left(T x_{2 n}, y_{2 n+1}\right)=d\left(y_{2 n}, y_{2 n+1}\right),
$$

and hence

$$
\phi\left(d\left(T x_{2 n}, S x_{2 n+1}\right)\right) \leq \phi\left(d\left(T x_{2 n}, y_{2 n+1}\right)\right)=\phi\left(d\left(y_{2 n}, y_{2 n+1}\right)\right) .
$$

Now, as in Case 1, we obtain

$$
\phi\left(d\left(T x_{2 n}, S x_{2 n+1}\right)\right) \leq(a+2 b) \phi\left(d\left(T x_{2 n}, S x_{2 n-1}\right)\right) .
$$

In case $\left(S x_{2 n-1}, T x_{2 n}\right) \in Q_{1} \times P_{\circ}$, then

$$
\phi\left(d\left(S x_{2 n-1}, T x_{2 n}\right)\right) \leq(a+2 b) \phi\left(d\left(S x_{2 n-1}, T x_{2 n-2}\right)\right) .
$$

Case 3. If $\left(T x_{2 n}, S x_{2 n+1}\right) \in P_{1} \times Q_{\circ}$, then $S x_{2 n-1} \in Q_{\circ}$. Proceeding as in Case 1, we get

$$
d\left(T x_{2 n}, S x_{2 n+1}\right)=d\left(T x_{2 n}, y_{2 n+1}\right) \leq d\left(T x_{2 n}, y_{2 n}\right)+d\left(y_{2 n}, y_{2 n+1}\right) .
$$


Note that $d\left(y_{2 n}, y_{2 n+1}\right)=d\left(F x_{2 n}, G x_{2 n-1}\right)$, therefore

$$
\begin{aligned}
\phi\left(d\left(y_{2 n}, y_{2 n+1}\right)\right) & =\phi\left(d\left(F x_{2 n}, G x_{2 n-1}\right)\right) \leq \\
& \leq(a+2 b) \phi\left(d\left(T x_{2 n}, S x_{2 n-1}\right)\right)<\phi\left(d\left(T x_{2 n}, S x_{2 n-1}\right)\right)
\end{aligned}
$$

thus $d\left(y_{2 n}, y_{2 n+1}\right) \leq d\left(T x_{2 n}, S x_{2 n-1}\right)$, as $\phi$ is an increasing function. Therefore, we can write

$$
d\left(T x_{2 n}, S x_{2 n+1}\right) \leq d\left(T x_{2 n}, y_{2 n}\right)+d\left(T x_{2 n}, S x_{2 n-1}\right)=d\left(S x_{2 n-1}, y_{2 n}\right)
$$

and hence

$$
\begin{aligned}
\phi\left(d\left(T x_{2 n}, S x_{2 n+1}\right)\right) & \leq \phi\left(d\left(S x_{2 n-1}, y_{2 n}\right)\right) \leq(a+2 b) \phi\left(d\left(T x_{2 n-2}, S x_{2 n-1}\right)\right) \leq \\
& \leq h \phi\left(d\left(T x_{2 n-2}, S x_{2 n-1}\right)\right), \quad \text { where } \quad h=(a+2 b) .
\end{aligned}
$$

Thus in all the cases, we have

$$
\phi\left(d\left(T x_{2 n}, S x_{2 n+1}\right)\right) \leq h \max \left\{\phi\left(d\left(S x_{2 n-1}, T x_{2 n}\right)\right), \phi\left(d\left(T x_{2 n-2}, S x_{2 n-1}\right)\right)\right\}
$$

whereas

$$
\phi\left(d\left(S x_{2 n+1}, T x_{2 n+2}\right)\right) \leq h \max \left\{\phi\left(d\left(S x_{2 n-1}, T x_{2 n}\right)\right), \phi\left(d\left(T x_{2 n}, S x_{2 n+1}\right)\right)\right\} .
$$

It can be shown by induction that for $n \geq 1$,

$$
\phi\left(d\left(T x_{2 n}, S x_{2 n+1}\right)\right)<k^{2 n-1} \max \left\{\phi\left(d\left(T x_{0}, S x_{1}\right)\right), \phi\left(d\left(S x_{1}, T x_{2}\right)\right)\right\}
$$

and

$$
\phi\left(d\left(S x_{2 n+1}, T x_{2 n+2}\right)\right)<k^{2 n} \max \left\{\phi\left(d\left(S x_{1}, T x_{2}\right)\right), \phi\left(d\left(T x_{0}, S x_{1}\right)\right)\right\} .
$$

Now, for any positive integer $p$, we have

$$
\begin{aligned}
& \phi\left(d\left(T x_{2 n}, S x_{2 n+p}\right)\right) \leq \\
\leq & \phi\left\{d\left(T x_{2 n}, S x_{2 n+1}\right)+d\left(S x_{2 n+1}, T x_{2 n+2}\right)+\cdots+d\left(T x_{2 n+p-1}, S x_{2 n+p}\right)\right\} \leq \\
\leq & \phi\left\{\left(1+k+k^{2}+\cdots+k^{p-1}\right) k^{2 n} \max \left[d\left(T x_{0}, S x_{1}\right), d\left(S x_{1}, T x_{2}\right)\right]\right\} \leq \\
\leq & \phi\left\{\left(\frac{k^{2 n}}{1-k}\right) \max \left[d\left(T x_{0}, S x_{1}\right), d\left(S x_{1}, T x_{2}\right)\right]\right\}
\end{aligned}
$$

which shows that the sequence

$$
\left\{T x_{0}, S x_{1}, T x_{2}, S x_{3}, \ldots, S x_{2 n-1}, T x_{2 n}, S x_{2 n+1}, \ldots\right\}
$$

is Cauchy. Then as noted in [10], there exists at least one subsequence $\left\{T x_{2 n_{k}}\right\}$ or $\left\{S x_{2 n_{k}+1}\right\}$ which is contained in $P_{\circ}$ or $Q_{\circ}$ respectively and finds its limit $z \in K$. Since $T K$ as well as $S K$ are closed subspaces of $X$ and $\left\{T x_{2 n_{k}}\right\}$ is Cauchy in $T K$, it converges to a point $z \in T K$. Let $v \in T^{-1} z$, then $T v=z$. Similarly $\left\{S x_{2 n_{k}+1}\right\}$ being a subsequence of Cauchy sequence

$$
\left\{T x_{0}, S x_{1}, T x_{2}, S x_{3}, \ldots, S x_{2 n-1}, T x_{2 n}, S x_{2 n+1}, \ldots\right\}
$$


also converges to $z$ as $S K$ is closed. Using (2.5.1), one can write

$$
\begin{aligned}
& \phi\left(d\left(F v, T x_{2 n_{k}}\right)\right)=\phi\left(d\left(F v, G x_{2 n_{k}-1}\right)\right) \leq \\
& \leq a \max \left\{\frac{1}{2} \phi\left(d\left(T v, S x_{2 n_{k}-1}\right)\right), \phi(d(T v, F v)), \phi\left(d\left(S x_{2 n_{k}-1}, G x_{2 n_{k}-1}\right)\right)\right\}+ \\
& \quad+b\left\{\phi\left(d\left(T v, G x_{2 n_{k}-1}\right)\right)+\phi\left(d\left(S x_{2 n_{k}-1}, F v\right)\right)\right\}
\end{aligned}
$$

which on letting $k \rightarrow \infty$, reduces to

$$
\phi(d(F v, z)) \leq(a+b) \phi(d(F v, z))
$$

yielding thereby $F v=z$. Thus, we get $F v=z=T v$ which shows that $v$ is a coincidence point of $(F, T)$.

Since $(F, T)$ is coincidentally commuting, therefore

$$
z=T v=F v \Rightarrow F z=F T v=T F v=T z .
$$

To prove that $z$ is a fixed point of $F$, consider

$$
\begin{aligned}
& \phi\left(d\left(F z, T x_{2 n_{k}}\right)\right)=\phi\left(d\left(F z, G x_{2 n_{k}-1}\right)\right) \leq \\
& \leq a \max \left\{\frac{1}{2} \phi\left(d\left(T z, S x_{2 n_{k}-1}\right)\right), \phi(d(T z, F z)), \phi\left(d\left(S x_{2 n_{k}-1}, G x_{2 n_{k}-1}\right)\right)\right\}+ \\
& +b\left\{\phi\left(d\left(T z, G x_{2 n_{k}-1}\right)\right)+\phi\left(d\left(S x_{2 n_{k}-1}, F z\right)\right)\right\}
\end{aligned}
$$

which on letting $k \rightarrow \infty$, reduces to

$$
\phi(d(F z, z)) \leq(a+b) \phi(d(F z, z))
$$

which shows that $z$ is a common fixed point of $F$ and $T$.

Further, since Cauchy sequence $\left\{T x_{2 n_{k}}\right\}$ converges to $z \in K$ and $z=F v$, $z \in F K \cap K \subset S K$, there exists $w \in K$ such that $S w=z$. Again, using (2.5.1), we get

$$
\begin{aligned}
& \phi(d(S w, G w))=\phi(d(z, G w))=\phi(d(F z, G w)) \leq \\
& \leq a \max \left\{\frac{1}{2} \phi(d(T z, S w)), \phi(d(T z, F z)), \phi(d(S w, G w))\right\}+ \\
& \quad+b\{\phi(d(T z, G w))+\phi(d(S w, F z))\}
\end{aligned}
$$

implying thereby $S w=G w$ which shows that $w$ is a coincidence point of $(G, S)$. Now, repeating the foregoing arguments, one can show that $z$ is a common fixed point of $(S, G)$.

In case $F K$ and $G K$ are closed subspaces, then $z \in F K \cap K \subseteq S K$ or $z \in$ $G K \cap K \subseteq T K$. The analogous arguments establish the coincidence point of $(F, T)$ as well as $(S, G)$. If we assume that there exists a subsequence $\left\{S x_{2 n_{k}+1}\right\}$ which is contained in $Q_{\circ}$ with $T K$ as well as $S K$ are closed subspaces of $X$, then proof can be completed on similar lines, hence it is omitted.

The uniqueness of a common fixed point of $F, T, G$ and $S$ follow easily. This completes the proof.

The following remarks are pertinent in respect of Theorem 3.1. 
Remark 3.1. By choosing F, G,T and $S$ suitably, one can derive a multitude of fixed point theorems for a pair and triode of mappings which are seeming new to the literature.

Remark 3.2. By setting $F=G, T=S=I_{K}$ and $\phi(t)=t$, one deduces a slightly improved version of a result due to Khan et al. [15].

Remark 3.3. By setting $F=G, T=S=I_{K}$ and $\phi(t)=t$, one deduces a result due to Khan and Bhardwaj [14].

Remark 3.4. By setting $F=G, S=T=I_{K}$ and $\phi(t)=t$ with $b=0$, one deduces a result for nonself mappings satisfying Bianchini [5] type condition.

Remark 3.5. By setting $F=G, S=T=I_{K}, \phi(t)=t$ with $a=0$, one deduces a result for self mappings satisfying Chatterjea [6] type condition provided $K=X$.

Now, we prove Theorem 3.1 for pointwise $R$-weakly commuting pairs where we require the continuity of all four maps.

Theorem 3.2. Let $(X, d)$ be a complete metrically convex metric space and $K$ a nonempty closed subset of $X$. Let $F, G, S, T: K \rightarrow X$ be the mappings which satisfy (2.5.1), (i) and (ii). Suppose that

(vi) $(F, T)$ and $(G, S)$ are pointwise $R$-weakly commuting pairs;

(vii) $F, T, G$ and $S$ are continuous on $K$.

Then $F, G, S$ and $T$ have a unique common fixed point.

Proof. Following the lines of the proof of Theorem 3.1, suppose that there is a subsequence $\left\{T x_{2 n_{k}}\right\}$ which is contained in $P_{\circ}$. Furthermore, subsequences $\left\{T x_{2 n_{k}}\right\}$ and $\left\{S x_{2 n_{k}+1}\right\}$ both converge to $z \in K$ as $K$ is a closed subset of complete metric space $(X, d)$. Since $T x_{2 n_{k}}=G x_{2 n_{k}-1}$ and $S x_{2 n_{k}-1} \in K$, using pointwise $R$-weak commutativity of $(G, S)$, we have

$$
d\left(S G x_{2 n_{k}-1}, G S x_{2 n_{k}-1}\right) \leq R_{1} d\left(G x_{2 n_{k}-1}, S x_{2 n_{k}-1}\right)
$$

for some $R_{1}>0$. Also

$$
d\left(S G x_{2 n_{k}-1}, G z\right) \leq d\left(S G x_{2 n_{k}-1}, G S x_{2 n_{k}-1}\right)+d\left(G S x_{2 n_{k}-1}, G z\right) .
$$

Making $k \rightarrow \infty$ in (3.2.1) and (3.2.2) and using continuity of $G$ and $S$, one gets $d(S z, G z) \leq 0$ yielding thereby $G z=S z$.

Since $y_{2 n_{k}+1}=F x_{2 n_{k}}$ and $T x_{2 n_{k}} \in K$, the pointwise $R$-weak commutativity of $(F, T)$ implies

$$
d\left(T F x_{2 n_{k}}, F T x_{2 n_{k}}\right) \leq R_{2} d\left(F x_{2 n_{k}}, T x_{2 n_{k}}\right)
$$

for some $R_{2}>0$. Also

$$
d\left(T F x_{2 n_{k}}, F z\right) \leq d\left(T F x_{2 n_{k}}, F T x_{2 n_{k}}\right)+d\left(F T x_{2 n_{k}}, F z\right) .
$$

Therefore, as previously using the continuity of $F$ and $T$, one gets $d(T z, F z) \leq 0$ giving thereby $T z=F z$ as $k \rightarrow \infty$. 
If we assume that there exists a subsequence $\left\{S x_{2 n_{k}+1}\right\}$ which is contained in $Q_{\circ}$, then analogous arguments establish the earlier conclusions. The rest of the proof is identical to that of Theorem 3.1, hence it is omitted.

If we utilize 'weak commutativity' instead of 'pointwise $R$-weak commutativity' in Theorem 3.2, then the continuity of any one of the mappings is required. In this regard the following holds.

Theorem 3.3. Let $(X, d)$ be a complete metrically convex metric space and $K$ a nonempty closed subset of $X$. Let $F, G, S, T: K \rightarrow X$ be the mappings which satisfy (2.5.1), (i) and (ii). Suppose that

(viii) $(F, T)$ and $(G, S)$ are weakly commuting pairs;

(ix) one of $F, G, S$ and $T$ is continuous on $K$.

Then $F, G, S$ and $T$ have a unique common fixed point.

Proof. Following the lines of the proof of the Theorem 3.1, we can show that the sequence $\left\{T x_{2 n}\right\}$ is Cauchy and there exists at least one subsequence $\left\{T x_{2 n_{k}}\right\}$ or $\left\{S x_{2 n_{k}+1}\right\}$ which is contained in $P_{\circ}$ or $Q_{\circ}$ respectively. Suppose that there exists a subsequence $\left\{T x_{2 n_{k}}\right\}$ which is contained in $P_{\circ}$ for each $k \in N$. Firstly, assume that $S$ is continuous on $K$, then due to continuity of $S,\left\{S T x_{2 n_{k}}\right\}$ converges to $S z$. On using weak commutativity of the pair $(G, S)$, one can write

$$
d\left(S T x_{2 n_{k}}, G S x_{2 n_{k}-1}\right) \leq d\left(S x_{2 n_{k}-1}, G x_{2 n_{k}-1}\right)
$$

which on letting $k \rightarrow \infty$, yields

$$
d\left(S z, G S x_{2 n_{k}-1}\right) \rightarrow 0 .
$$

In order to show that $S z=z$, consider

$$
\begin{aligned}
\phi\left(d\left(F x_{2 n_{k}}, G S x_{2 n_{k}-1}\right)\right) \leq \\
\leq a \max \left\{\frac{1}{2} \phi\left(d\left(T x_{2 n_{k}}, S S x_{2 n_{k}-1}\right)\right), \phi\left(d\left(T x_{2 n_{k}}, F x_{2 n_{k}}\right)\right),\right. \\
\left.\phi\left(d\left(S S x_{2 n_{k}-1}, G S x_{2 n_{k}-1}\right)\right)\right\}+b\left\{\phi\left(d\left(T x_{2 n_{k}}, G S x_{2 n_{k}-1}\right)\right)+\right. \\
\left.+\phi\left(d\left(S S x_{2 n_{k}-1}, F x_{2 n_{k}}\right)\right)\right\}
\end{aligned}
$$

which on letting $k \rightarrow \infty$, reduces to

$$
\begin{aligned}
\phi(d(z, S z)) & \leq a \max \left\{\frac{1}{2} \phi(d(z, S z)), 0,0\right\}+b\{\phi(d(z, S z))+\phi(d(z, S z))\} \leq \\
& \leq\left(\frac{a}{2}+2 b\right) \phi(d(z, S z)),
\end{aligned}
$$

yielding thereby $z=S z$. Next, we consider

$$
\begin{array}{r}
\phi\left(d\left(F x_{2 n_{k}}, G z\right)\right) \leq a \max \left\{\frac{1}{2} \phi\left(d\left(T x_{2 n_{k}}, S z\right)\right), \phi\left(d\left(T x_{2 n_{k}}, F x_{2 n_{k}}\right)\right), \phi(d(S z, G z))\right\}+ \\
+b\left\{\phi\left(d\left(T x_{2 n_{k}}, G z\right)\right)+\phi\left(d\left(S z, F x_{2 n_{k}}\right)\right)\right\}
\end{array}
$$


which on letting $k \rightarrow \infty$, reduces to

$$
\begin{aligned}
\phi(d(z, G z)) & \leq a \max \{0,0, \phi(d(z, G z))\}+b\{\phi(d(z, G z))+\phi(d(z, G z))\} \leq \\
& \leq(a+2 b) \phi(d(z, G z)),
\end{aligned}
$$

implying thereby $z=G z$.

Since $z$ is in the range of $G$ and due to the relation $G K \cap K \subset T K$, there exists a point $u \in K$ such that $T u=z$. Therefore

$$
\begin{aligned}
\phi(d(F u, z))= & \phi(d(F u, G z)) \\
\leq & a \max \left\{\frac{1}{2} \phi(d(T u, S z)), \phi(d(T u, F u)), \phi(d(S z, G z))\right\}+ \\
& \quad+b\{\phi(d(T u, G z))+\phi(d(S z, F u))\} \leq \\
\leq & a \max \{0, \phi(d(z, F u)), 0\}+b\{0+\phi(d(z, F u))\} \leq \\
\leq & (a+b) \phi(d(z, F u)),
\end{aligned}
$$

implying thereby $F u=z=T u$. Also, we can write

$$
d(F z, T F u)=d(F T u, T F u) \leq d(F u, T u)=0,
$$

which implies that $F z=T z$. In order to show that $F z=z$, we consider

$$
\begin{aligned}
\phi(d(F z, z))= & \phi(d(F z, G z)) \\
\leq & a \max \left\{\frac{1}{2} \phi(d(T z, S z)), \phi(d(T z, F z)), \phi(d(S z, G z))\right\}+ \\
& \quad+b\{\phi(d(T z, G z))+\phi(d(S z, F z))\} \leq \\
\leq & a \max \left\{\frac{1}{2} \phi(d(F z, z)), 0,0\right\}+b\{\phi(d(F z, z))+\phi(d(z, F z))\} \leq \\
\leq & \left(\frac{a}{2}+2 b\right) \phi(d(z, F z)),
\end{aligned}
$$

yielding thereby $F z=z=T z$.

Hence $S z=G z=F z=T z=z$. Thus $z$ is a common fixed point of $F, G, S$ and $T$. In case $T$ is continuous, a similar proof can be outlined, hence it is omitted.

Next, let us assume that $F$ is continuous, then the sequence $\left\{F T x_{2 n_{k}}\right\}$ converges to $F z$. Since the pair $(F, T)$ commutes weakly, therefore as earlier it follows that $\left\{T F x_{2 n_{k}}\right\}$ converges to $F z$. Now, consider

$$
\begin{aligned}
& \phi\left(d\left(F F x_{2 n_{k}}, G x_{2 n_{k}-1}\right)\right) \leq a \max \left\{\frac{1}{2} \phi\left(d\left(T F x_{2 n_{k}}, S x_{2 n_{k}-1}\right)\right),\right. \\
& \left.\phi\left(d\left(T F x_{2 n_{k}}, F F x_{2 n_{k}}\right)\right), \phi\left(d\left(S x_{2 n_{k}-1}, G x_{2 n_{k}-1}\right)\right)\right\}+ \\
& +b\left\{\phi\left(d\left(T F x_{2 n_{k}}, G x_{2 n_{k}-1}\right)\right)+\phi\left(d\left(S x_{2 n_{k}-1}, F F x_{2 n_{k}}\right)\right)\right\}
\end{aligned}
$$

which on letting $k \rightarrow \infty$, reduces to

$$
\phi(d(z, F z)) \leq\left(\frac{a}{2}+2 b\right) \phi(d(F z, z))
$$


yielding thereby $F z=z$. Similarly

$$
\begin{aligned}
& \phi\left(d\left(T z, G x_{2 n_{k}-1}\right)\right) \leq \\
& \leq a \max \left\{\frac{1}{2} \phi\left(d\left(T z, S x_{2 n_{k}-1}\right)\right), \phi(d(T z, F z)), \phi\left(d\left(S x_{2 n_{k}-1}, G x_{2 n_{k}-1}\right)\right)\right\}+ \\
& +b\left\{\phi\left(d\left(T z, G x_{2 n_{k}-1}\right)\right)+\phi\left(d\left(S x_{2 n_{k}-1}, F z\right)\right)\right\}
\end{aligned}
$$

which on letting $k \rightarrow \infty$, reduces to

$$
\phi(d(T z, z)) \leq(a+b) \phi(d(T z, z)),
$$

a contradiction, implying thereby $T z=z$.

Note as earlier $F z=z$, it means that $z$ is in the range of $F$ and then due to the relation $F K \cap K \subset S K$, there exists a point $v \in K$ such that $S v=z$.

Now consider

$$
\begin{aligned}
\phi(d(z, G v)) & =\phi(d(F z, G v)) \\
\leq & a \max \left\{\frac{1}{2} \phi(d(T z, S v)), \phi(d(T z, F z)), \phi(d(S v, G v))\right\}+ \\
& \quad+b\{\phi(d(T z, G v))+\phi(d(S v, F z))\} \leq \\
\leq & (a+b) \phi(d(z, G v)),
\end{aligned}
$$

yielding thereby $z=G v$. Since the pair $(G, S)$ commutes weakly, therefore

$$
d(G S v, S G v) \leq d(G v, S v)=0,
$$

implying thereby $G S v=S G v$ which implies that $G z=S z$.

In order to prove $G z=z$, consider

$$
\begin{aligned}
\phi(d(z, G z)) & =\phi(d(F z, G z)) \\
& \leq a \max \left\{\frac{1}{2} \phi(d(T z, S z)), \phi(d(T z, F z)), \phi(d(S z, G z))\right\}+ \\
& \quad+b\{\phi(d(T z, G z))+\phi(d(S z, F z))\} \leq \\
& \leq(a+b) \phi(d(z, G z)),
\end{aligned}
$$

which implies that $z=G z$. Thus we obtain $S z=z=T z=G z=F z$, hence $z$ is a common fixed point of $F, G, S$ and $T$. If we assume $G$ to be continuous, a similar proof can be outlined, hence it is omitted. If $\left\{S x_{2 n_{k}+1}\right\}$ is contained in $Q_{\circ}$, then the proof goes on similar lines, hence it is also omitted.

The uniqueness of the common fixed point follows easily due to contraction condition (2.5.1). This completes the proof.

Remark 3.6. Remarks 3.1-3.5 remain pertinent in the context of Theorem 3.3.

\section{An Illustrative Example}

Finally, we furnish an example to establish the utility of our results over earlier ones especially those contained in Khan et al. [15], Khan and Bhardwaj [14], Bianchini [5], Chatterjea [6] and others. 
Example 4.1. Let $X=[1, \infty)$ with Euclidean metric, $K=[1,3]$ and $\phi(t)=\sqrt{t}$. Define $F, G, S, T: K \rightarrow X$ as

$$
\begin{aligned}
& F x=\left\{\begin{array}{ll}
\frac{x+1}{2}, & \text { if } 1<x \leq 2 \\
1, & \text { if } x \in(2,3] \cup\{1\}
\end{array} \quad T x= \begin{cases}2 x^{2}-1, & \text { if } 1<x \leq 2 \\
1, & \text { if } x \in(2,3] \cup\{1\}\end{cases} \right. \\
& G x=\left\{\begin{array}{ll}
\frac{x^{2}+1}{2}, & \text { if } 1<x \leq 2 \\
1, & \text { if } x \in(2,3] \cup\{1\}
\end{array} \text { and } \quad S x= \begin{cases}2 x^{4}-1, & \text { if } 1<x \leq 2 \\
1, & \text { if } x \in(2,3] \cup\{1\} .\end{cases} \right.
\end{aligned}
$$

Notice that $\delta K$ (the boundary of $K$ ) $=\{1,3\}$. Clearly $T K \cap S K=[1,7] \cap$ $[1,31]=[1,7]$ and hence $\delta K=\{1,3\} \subset T K \cap S K$. Further $F K \cap K=[1,1.5] \cap$ $[1,3]=[1,1.5] \subset S K$ and $G K \cap K=[1,2.5] \cap[1,3]=[1,2.5] \subset T K$.

Also

$$
\begin{aligned}
& T 1=1 \in \delta K \Rightarrow F 1=1 \in K, \\
& S 1=1 \in \delta K \Rightarrow G 1=1 \in K, \\
& T 3=1 \in \delta K \Rightarrow F 3=1 \in K, \\
& S 3=1 \in \delta K \Rightarrow G 3=1 \in K .
\end{aligned}
$$

Moreover, if $1<x, y \leq 2$, then

$$
\begin{aligned}
\phi(d(F x, G y)) & =\sqrt{\frac{\left|x-y^{2}\right|}{2}}=\frac{\sqrt{\left|x-y^{2}\right|} \sqrt{\left|x+y^{2}\right|}}{\sqrt{2} \sqrt{\left|x+y^{2}\right|}}= \\
& =\frac{\sqrt{\left|x^{2}-y^{4}\right|}}{\sqrt{2} \sqrt{\left|x+y^{2}\right|}}=\frac{1}{\sqrt{\left|x+y^{2}\right|}}\left(\frac{1}{2} \phi(d(T x, S y))\right) \leq \\
& \leq \frac{1}{\sqrt{2}}\left(\frac{1}{2} \phi(d(T x, S y))\right) .
\end{aligned}
$$

Finally, if $1<x \leq 2$ and $y \in(2,3] \cup\{1\}$, then

$$
\begin{aligned}
\phi(d(F x, G y)) & =\sqrt{\frac{|x-1|}{2}}=\frac{\sqrt{|x-1|} \sqrt{|x+1|}}{\sqrt{2} \sqrt{|x+1|}}= \\
& =\frac{1}{\sqrt{|x+1|}}\left(\frac{1}{2} \phi(d(T x, S y))\right) \leq \\
& \leq \frac{1}{\sqrt{2}}\left(\frac{1}{2} \phi(d(T x, S y))\right) .
\end{aligned}
$$

Therefore condition (2.5.1) is satisfied if one chooses $a=\frac{1}{\sqrt{2}}$ and $b=\frac{1}{7}$. Moreover 1 is a point of coincidence as $T 1=F 1$ and $S 1=G 1$ whereas both the pairs $(F, T)$ and $(G, S)$ are coincidentally commuting as $T F 1=1=F T 1$, and $S G 1=1=$ $G S 1$. Also $F K, T K, G K$ and $S K$ are closed in $X$. Thus all the conditions of the Theorem 3.1 are satisfied and ' 1 ' is the unique common fixed point of $F, G$, $S$ and $T$.

Note that the mappings satisfying (2.5.1) need not satisfy (1.1.1) one by one. To substantiate this, consider the mapping $T$ with $1<x \leq 2$ and $y \in(2,3] \cup\{1\}$ 
then using (1.1.1), one gets

$$
\begin{aligned}
d(T x, T y) & =\left|2 x^{2}-2\right| \leq \\
& \leq a \max \left\{\left|x-2 x^{2}+1\right|,|y-1|\right\}+b\left\{|x-1|+\left|y-2 x^{2}+1\right|\right\}
\end{aligned}
$$

at $x=2$ and $y=2.5$ then

$$
\begin{aligned}
|8-2| & \leq a \max \{|2-8+1|,|2.5-2|\}+b\{|2-1|+|2.5-8+1|\} \\
6 & <5 a+\left(\frac{1-a}{2}\right) 5.5
\end{aligned}
$$

implying thereby $1<a$, a contradiction, which establishes the utility of the results proved in this paper. Thus Theorem 1.1 cannot be used in the context of mapping $T$.

Acknowledgement. The authors are grateful to the learned referee for his critical reading of the entire manuscript and suggesting many improvements. The first author is also grateful to the university grant commission in India for the financial assistance (Project No: F.30-246/2004(SR)).

\section{REFERENCES}

[1] N.A. Assad and W.A. Kirk, Fixed point theorems for set-valued mappings of contractive type, Pacific J. Math., 43(3) (1972), 553-562.

[2] N.A. Assad, On some nonself nonlinear contractions, Math. Japon., 33(1) (1988), 17-26.

[3] N.A. Assad, On some nonself mappings in Banach spaces, Math. Japon., 33(4) (1988), $501-515$.

[4] A.J. Assad and Zaheer Ahmad, Common fixed point of a pair of mappings in Banach spaces, Southeast Asian Bull. Math., 23 (1999), 349-355.

[5] R.M.T. Bianchini, Su un problema dis. Reich riguardante lateoria dei punti fissi, Boll. Un. Mat. Ital., 5 (1972), 103-108.

[6] S.K. Chatterjea, Fixed point theorems, C.R. Acad. Bulgare Sci., 25 (1972), 727-730.

[7] D. Delbosco, Un Estensione Di Un Teorema Sul Punto. Fisso D. Reich, Rend. Sem. Mat. Univers. Politecn. Torino., 35 (1976-77), 323-329.

[8] B. Fisher, A fixed point mapping, Bull. Calcutta Math. Soc., 68 (1976), 265-266.

[9] O. Hadžić, On coincidence points in convex metric spaces, Univ. u Novom Sadu, Zb. Rad. Prirod. Mat. Fak. Ser. Mat., 19(2) (1989), 233-240.

[10] O. Hadžić and Lj. Gajic, Coincidence points for set-valued mappings in convex metric spaces, Univ. u Novom. Sadu., Zb. Rad. Prirod. Mat. Fak. Ser. Mat., 16(1) (1986), 13-25.

[11] M. Imdad and S. Kumar, Boyd and Wong type theorems for two pairs of non self mappings, Nonlinear Analysis Forum, 8(1) (2003), 69-78.

[12] G. Jungck, Compatible mappings and common fixed points, Internat. J. Math. Math. Sci., 9(4) (1986), 771-779.

[13] G. Jungck and B.E. Rhoades, Fixed points for set-valued functions without continuity, Indian J. Pure Appl. Math., 29(3) (1998), 227-238. 
[14] M.D. Khan and R. Bharadwaj, A fixed point theorem in metrically convex space, Indian J. Math., 43(3) (2001), 373-379.

[15] M.S. Khan, H.K. Pathak and M.D. Khan, Some fixed point theorems in metrically convex spaces, Georgian Math. J., 7(3) (2000), 523-530.

[16] M.S. Khan, M. Swaleh and S. Sessa, Fixed point theorems by altering distances between the points, Bull. Austral. Math. Soc., 30 (1984), 1-9.

[17] R.P. Pant, Common fixed points of noncommuting mappings, J. Math. Anal. Appl., 188 (1994), 436-440.

[18] E. Rakotch, A note on contractive mapping, Proc. Amer. Math. Soc., 13 (1962), 459-465.

[19] S. Reich, Kannan's fixed point theorem, Bull. U. M. I., 4 (1971), 1-11.

[20] S. Sessa, On a weak commutativity condition in fixed point considerations, Publ. Inst. Math., 32(46) (1982), 149-153.

[21] F. Skof, Teorema, di. Punti. Fisso. Per. Applicazioni Negli Spazi Metrici, Atti Acad. Sci. Tornio., III (1977), 323-329.

Department of Mathematics, Aligarh Muslim University, Aligarh 202002 INDIA

E-mail address: mhimdad@yahoo.co.in

Department of Mathematics, Aligarh Muslim University, Aligarh 202002

INDIA

E-mail address: k_ladlay@yahoo.com 